\title{
Reliability and factor structure of the Hospital Anxiety and Depression Scale in a polytrauma clinic
}

\author{
Laura Boxley, PhD; ${ }^{1}$ Jennifer M. Flaherty, PhD; ${ }^{2 *}$ Robert J. Spencer, PhD; ${ }^{3}$ Lauren L. Drag, PhD; ${ }^{4}$ Percival H. \\ Pangilinan, MD; $;^{3,5}$ Linas A. Bieliauskas, $\mathbf{P h D}^{3,5}$ \\ ${ }^{1}$ Department of Psychiatry and Behavioral Health, The Ohio State University Wexner Medical Center, Columbus, OH; \\ ${ }^{2}$ Department of Psychiatry \& Behavioral Sciences, Eastern Virginia Medical School, Norfolk, VA; ${ }^{3}$ Department of \\ Veterans Affairs Ann Arbor Healthcare System, Ann Arbor, MI; ${ }^{4}$ Department of Neurology and Neurological Sciences, \\ Stanford University School of Medicine, Palo Alto, CA; ${ }^{5}$ University of Michigan Health System, University of Michigan, \\ Ann Arbor, MI
}

\begin{abstract}
The Hospital Anxiety and Depression Scale (HADS) is a brief self-report measure of anxiety and depression symptoms. This study examined the internal consistency and factor structure of the HADS among Veterans in a polytrauma/traumatic brain injury clinic. The sample consisted of 312 Veterans. A confirmatory factor analysis of the depression and anxiety subscales showed, not surprisingly, that the two factors were highly correlated $(r=0.70)$. Goodness of fit statistics for the two-factor model were acceptable (root mean square error of approximation $=0.06$, comparative fit index $=$ $0.94)$. The HADS demonstrated very good reliability overall $($ alpha $=0.89)$ and for the individual subscales (alpha $=0.84)$. This study supports the use of the HADS as a screen for depression and anxiety in the assessment of mild traumatic brain injury in a Veteran population.
\end{abstract}

Key words: anxiety, concussion, confirmatory factor analysis, depression, factor structure, internal consistency, mild traumatic brain injury, polytrauma, reliability, self-report.

\section{INTRODUCTION}

Because of overlapping symptoms and comorbid conditions, when assessing soldiers and Veterans with suspected traumatic brain injury (TBI) we should also assess other conditions. Although a comprehensive evaluation of all psychiatric conditions is not usually practical in a time-limited TBI evaluation, we should screen for conditions that commonly co-occur with or mimic symptoms of TBI. Among the most commonly encountered of these conditions are anxiety and depression [13]. This study examines the psychometric properties of a brief self-report measure of anxiety and depression, the

Abbreviations: $\mathrm{BAI}=$ Beck Anxiety Inventory, BDI-2 $=$ Beck Depression Inventory-II, CFA = confirmatory factor analysis, $\mathrm{CFI}=$ comparative fit index, HADS $=$ Hospital Anxiety and Depression Scale, HADS-A = Hospital Anxiety and Depression Scale anxiety items, HADS-D = Hospital Anxiety and Depression Scale depression items, HAM-D $=$ Hamilton Depression Rating Scale, mTBI = mild traumatic brain injury, PTSD $=$ posttraumatic stress disorder, RMSEA = root mean square error of approximation, SCID-IV = Structured Clinical Interview for the Diagnostic and Statistical Manual of Mental Disorders-Fourth Edition, TBI = traumatic brain injury, VA = Department of Veterans Affairs.

*Address all correspondence to Jennifer M. Flaherty, PhD; EVMS Neuropsychology Program, Andrews Hall, Ste 461, 721 Fairfax Ave, Norfolk, VA 23507; 757-446-8400;

fax: 757-446-8401. Email: flaherjm@evms.edu

http://dx.doi.org/10.1682/JRRD.2015.05.0088 
Hospital Anxiety and Depression Scale (HADS) [4], in a sample of Veterans evaluated in a Department of Veterans Affairs (VA) polytrauma/TBI clinic. The psychometric measures of the HADS have not been previously investigated in this sample.

The HADS is among several brief measures that have been developed to screen for anxiety and depression. Other, more widely used instruments include the Beck Anxiety Inventory (BAI) [5], the Beck Depression Inventory-II (BDI-2) [6], and the Hamilton Depression Rating Scale (HAM-D) [7]. Brief self-report measures can be useful for quantifying symptoms at a single point in time or tracking changes in symptoms over time. Although such instruments were not developed to diagnose psychiatric conditions, they are often used to supplement other assessment methods in settings where there is an emphasis on brevity. Both the BAI and BDI-2 are 21-item inventories that assess physical, affective, and cognitive symptoms of anxiety and depression. The BAI takes approximately $5 \mathrm{~min}$ to complete, and the BDI- 2 takes approximately 5 to $10 \mathrm{~min}$. Internal consistency of these measures is very good to excellent, ranging from 0.84 to 0.94 [8-10]. The HAM-D is a 17- to 29-item clinicianadministered test that focuses on the cognitive and affective aspects of depression. Internal consistency of the HAM-D is adequate to very good, ranging from 0.77 to 0.81 [11]. Although each of these measures has clinical utility, each has weaknesses that are not apparent in the HADS.

The HADS was developed in 1983 as a brief, portable self-report measure of anxiety and depression [4]. Because the HADS was intended for use with medical patients among whom physical problems are common, the items of the HADS deemphasize physical or somatic symptoms, such as decreased energy, and emphasize cognitive symptoms. Unlike the HAM-D, the HADS addresses both depression and anxiety and does not require a clinician for administration. The HADS has fewer items (i.e., 14) than the aforementioned measures and thus takes less time for patients to complete, approximately $5 \mathrm{~min}$. Scoring the HADS is simple, and similarly to the aforementioned measures, takes just a few minutes. The HADS is also freely available within the public domain. These features make the HADS attractive to clinicians and researchers who need a rapid, efficient assessment of psychiatric symptoms when there is not enough time to administer multiple, more comprehensive, or time-consuming instruments, such as the BAI, BDI-2, and the HAM-D [5-7].

The HADS is straightforward and requires minimal time for administration and scoring, but, as with any assessment instrument, its utility rests with its psychometric properties. The anxiety and depression subscales of the HADS have been found to have at least adequate internal consistency, with reliability estimates exceeding 0.70 among nondiagnosed individuals [12-13], medical patients [14-23], and psychiatric patients [24-25], which is similar to reliability estimates reported for the BAI, BDI-2, and HAM-D. Nevertheless, this instrument has not been as thoroughly studied in a head injury population. Factor analytic studies have examined whether the items of the HADS separate into distinct factors. Flint and Rifat used principle components analysis to determine that the HADS separated into two distinct factors in older patients with major depression [25]. A review of 747 papers reporting on studies of samples from mostly hospital clinics, but also samples from the general population, general practice, and psychiatric patients, found that most demonstrated the validity of the HADS using a two-factor solution, mainly principle components analysis, consisting of HADS anxiety items (HADS-A) and HADS depression items (HADS-D) [26]. The Cronbach alpha for HADS-A ranged from 0.68 to 0.93 (below adequate to excellent) with a mean of 0.83 (very good). Cronbach alpha for HADS-D ranged from 0.67 to 0.90 (below adequate to excellent) with a mean of 0.82 (very good). These coefficients are similar to those reported for the BDI-2, BAI, and HAM-D. Included in the review was a confirmatory factor analysis (CFA) by Dunbar et al. who tested different models and found that a three-factor model provided the closest fit across three age groups (18-19, 39-40, and 58-59 yr old) [27]. However, a twofactor model also showed goodness of fit statistics that were similar to the three-factor model. Measures of goodness of fit for the two-factor model were acceptable as follows: comparative fit index (CFI) ranging from 0.92 to 0.94, and root mean square error of approximation (RMSEA) ranging from 0.04 to 0.06 .

Several studies have confirmed the diagnostic validity of the HADS. In perhaps the most pertinent study to the current investigation, Whelan-Goodinson et al. examined the diagnostic validity of the HADS among patients with mild to severe TBIs using the Structured Clinical Interview for the Diagnostic and Statistical Manual of Mental Disorders-Fourth Edition (SCID-IV) as a criterion 
measure for anxiety and depression [28]. Depending on whether mild or severe cut offs were employed, 57 to 92 percent of those scoring in the anxious range on the HADS-A were determined to have an anxiety disorder according to the SCID-IV. Conversely, 72 to 83 percent of the sample scoring in the nonclinical range on the HADS-A did not meet SCID-IV criteria for an anxiety disorder. Similar results were obtained with the HADSD: 81 to 100 percent of participants who endorsed depression on the HADS-D met criteria for depression and 69 to 82 percent of individuals scoring in the nonclinical range on the HADS-D did not meet criteria for depression. Similar diagnostic validity statistics have been found in samples of patients with medical disorders [22,29-33] and mixed psychiatric disorders [21,34].

The current study seeks to evaluate the utility of the reliability and factor structure of the HADS among Veterans in a polytrauma/TBI clinic. This undertaking is important because an increasing number of Veterans are reporting postconcussive symptoms. Given the significant overlap between postconcussive symptoms and psychiatric symptoms, brief screening for psychiatric distress is a necessary part of a thorough mild TBI (mTBI) evaluation. First, the HADS will be subjected to a CFA to see if the items can be separated into distinct scales of depression and anxiety. Second, the existing anxiety and depression scales will be examined using the Cronbach alpha to evaluate internal consistency. A statistic exceeding 0.70 will be deemed adequate for a brief screening instrument, values around 0.80 will be very good, and values of 0.90 and above will be excellent [35-36].

\section{METHODS}

\section{Participants}

We conducted a retrospective review of deidentified data from Veterans who underwent neuropsychological testing as part of a comprehensive polytrauma/TBI evaluation. The majority of Veterans were referred to the TBI clinic after screening positive for a possible TBI using a standard VA clinical reminder that broadly assesses risk factors and symptoms of a head injury. Most individuals had not previously received mental health care. A total of 312 Veterans completed the HADS as part of neuropsychological testing, and 23 of these Veterans were seeking compensation for suspected mTBI that occurred during their military service. Data from many of these partici- pants have been previously reported in research examining self-reported cognitive symptoms and performance validity of a digit span test and a Rey Fifteen-Item Memory Test [37-40]. Participants were excluded if they sustained a head injury of more than mild severity (i.e., greater than $30 \mathrm{~min}$ loss of consciousness, greater than $24 \mathrm{~h}$ posttraumatic amnesia) or presented non-TBI central nervous system pathology, such as stroke or epilepsy. Participants over $50 \mathrm{yr}$ of age were excluded from the study because the majority of the Veterans evaluated in the clinic are younger Veterans returning from the recent conflicts in Iraq and Afghanistan, so we wanted to limit our investigation to this population. Additionally, we wanted to exclude the possible confounding effect of neurodegenerative disorders. The mean age was $29.2 \mathrm{yr}$ $( \pm 7.0)$, with $12.8 \mathrm{yr}( \pm 1.4)$ of education. Six individuals did not report their years of completed education. The sample was predominantly male (95.5\%).

\section{Measures}

The HADS consists of 14 items: 7 designed to measure depression and 7 designed to measure anxiety [4]. Items are rated on a Likert-type scale from 0 to 3. Total scores are calculated for the depression and anxiety items. Scores of 8 to 10 indicate mild symptoms, scores of 11 to 14 indicate moderate symptoms, and scores of 15 or higher indicate severe symptoms. Reliability of the total scale as well as the HADS-A and HADS-D subscales were assessed.

\section{Procedure}

The HADS was administered as part of a larger screening battery. Participants consisted of consecutive referrals to the polytrauma/TBI clinic as well as deidentified archived data, which was analyzed retrospectively. Following completion of the HADS and other measures, the other required components of the evaluation were completed, including a thorough clinical history, psychosocial needs assessment/psychoeducation, and neurologic examination. If clinically significant mental health symptoms were present during the evaluation and the participants were interested, they were referred to the relevant clinic for further mental health assessment and/or treatment.

\section{Statistical Analysis}

Statistical analyses were computed using IBM SPSS Statistics, Windows, Version 22 (IBM Corp; Armonk, New York) The data were screened for univariate and 
multivariate normality, linearity, and homoscedasticity. These analyses showed that univariate assumptions were met. The means, standard deviations, skewness, and kurtosis statistics for the 14 items are presented in the Table. Mahalanobis distance and Mardia coefficient of multivariate kurtosis were calculated to assess multivariate normality. Mardia coefficient $=13.5$, which exceeds the critical ratio of 5.6 indicating a violation of multivariate normality. Mahalanobis distance exceeded the chi-square of $27.7(d f=13), \alpha=0.001$ for 15 cases. These cases did not appear to be outliers and were retained for further analyses. A Bollen-Stine bootstrapping technique was employed to account for the violation of multivariate normality, and the results were only slightly different from using maximum likelihood estimation. Because maximum likelihood estimation has been shown to be relatively robust to violations of normality with large samples with no missing data and Bollen-Stine bootstrapping does not include adjusted goodness of fit measures, results are presented using maximum likelihood estimation [41]. A CFA was conducted to determine whether the two-factor structure holds in the current sample. Although there is no single criterion for assessing goodness of fit, general convention suggests the RMSEA should be less than 0.08 for acceptable fit and less than 0.05 for excellent fit [42-43]. The CFI should be greater than 0.90 for acceptable fit and greater than 0.95 for close

Table.

Means, standard deviations, skewness, and kurtosis of Hospital Anxiety and Depression Scale items.

\begin{tabular}{rcccc}
\hline Item & Mean & $\begin{array}{c}\text { Standard } \\
\text { Deviation }\end{array}$ & Skewness & Kurtosis \\
\hline 1 & 2.04 & 0.86 & -0.41 & -0.78 \\
2 & 1.43 & 0.99 & 0.30 & -0.96 \\
3 & 1.73 & 0.99 & -0.27 & -0.96 \\
4 & 0.94 & 0.79 & 0.30 & -0.81 \\
5 & 2.01 & 0.87 & -0.47 & 0.60 \\
6 & 1.23 & 0.73 & 0.01 & -0.45 \\
7 & 1.73 & 0.71 & -0.22 & -0.06 \\
8 & 1.65 & 0.90 & -0.00 & -0.84 \\
9 & 1.17 & 0.86 & -0.43 & -0.37 \\
10 & 1.12 & 0.92 & 0.28 & -0.91 \\
11 & 1.94 & 0.87 & -0.41 & -0.60 \\
12 & 1.33 & 0.97 & 0.15 & -0.97 \\
13 & 1.47 & 0.90 & 0.06 & -0.77 \\
14 & 1.16 & 1.05 & 0.57 & -0.86 \\
\hline \hline
\end{tabular}

fit [44]. Internal consistency of the HADS total scale, HADS-D, and HADS-A was examined using the Cronbach alpha.

\section{RESULTS}

The range of possible scores for each subscale is 0 to 21 , with a total possible 42 points. Scores of 8 to 10 indicate mild symptoms, scores of 11 to 14 indicate moderate symptoms, and scores of 15 or higher indicate severe symptoms. The mean score on the anxiety scale for this sample was $12.09( \pm 4.31)$, which is in the moderate range. Scores for $48(15.38 \%)$ individuals were not clinically meaningful. Scores for $66(21.15 \%)$ individuals indicated mild symptoms of anxiety, scores for 91 (29.17\%) individuals indicated moderate symptoms, and scores for 107 (34.29\%) individuals indicated severe symptoms.

The mean score on the depression scale for this sample was $8.87( \pm 4.54)$, which is in the mild range. Scores for $119(38.14 \%)$ individuals were not clinically meaningful. Scores for $83(26.60 \%)$ individuals indicated mild symptoms of depression, scores for 75 (24.04\%) individuals indicated moderate symptoms, and scores for $35(11.22 \%)$ individuals indicated severe symptoms.

Research and clinical practice have shown that anxiety and depression are related, have overlapping features, and often co-occur, so the two latent factors of anxiety and depression were allowed to correlate in the CFA. The model is depicted in the Figure. All of the paths in the model were statistically significant. The model chisquare was statistically significant, indicating that the model's predictions significantly deviated from the actual data pattern, $\chi^{2}(76)=168.85, p<0.001$. The RMSEA fit statistic was acceptable (RMSEA $=0.06)$, and the 90 percent confidence interval ranged from 0.05 to 0.08 . The CFI was also acceptable $(\mathrm{CFI}=0.94)$.

As expected, the two factors were highly correlated $(r=0.70)$. All of the paths from the latent to the observed variables were significant. The total HADS, HADS-A, and HADS-D all had very good reliabilities, the Cronbach alpha is 0.89 (approaching excellent) for the total scale and 0.84 for each of the subscales.

\section{DISCUSSION}

This study focused on determining the psychometric properties of the HADS in Veterans undergoing evaluation 


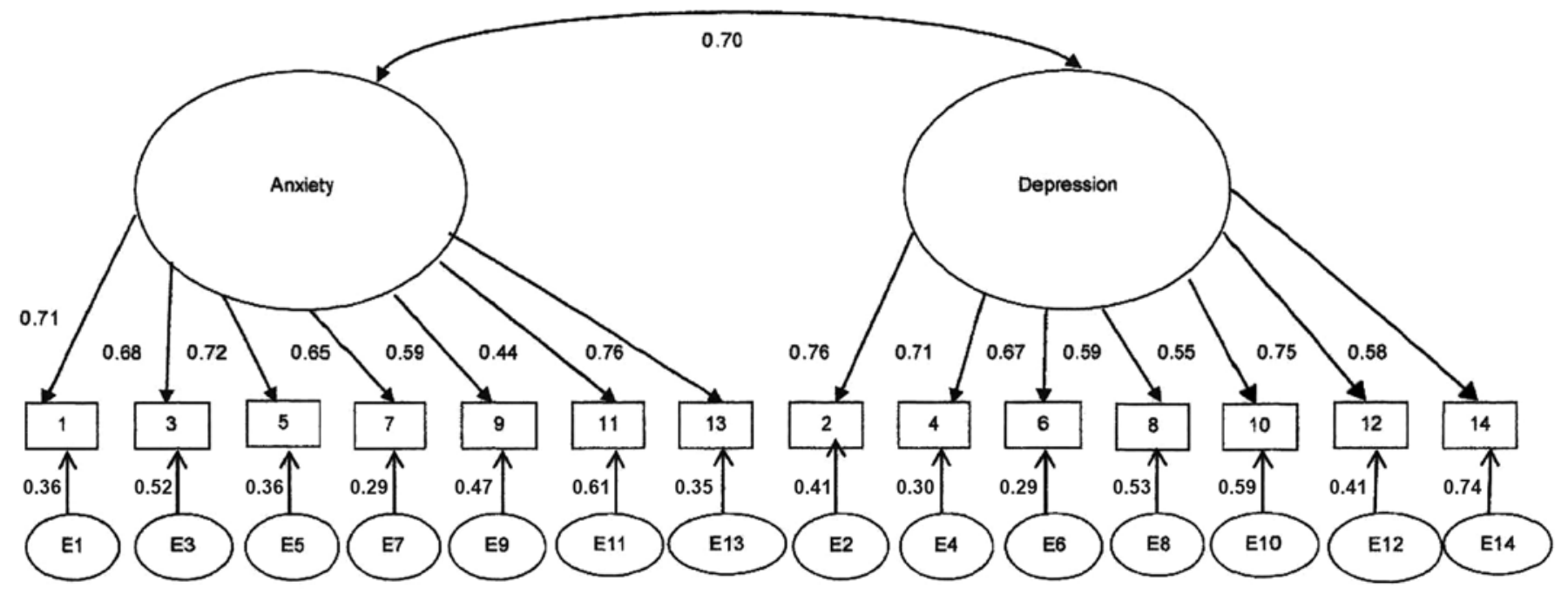

Figure.

Factor structure of the Hospital Anxiety and Depression Scale. Large ovals have latent variables. Item numbers in boxes are observed variables. Numbers next to arrows from latent to observed variables are the standardized estimated factor loadings. Numbers next to arrows from observed variables to error terms (E) are residual variances. Double-headed arrow indicates correlation between latent variables (anxiety and depression; 0.70).

in a polytrauma/TBI clinic. The results showed that the two-factor structure of the HADS holds in a sample of Veterans with suspected mTBI. The model fit statistics obtained in the current study were within the ranges found by Dunbar et al. in their two-factor models [27]. Although there was significant correlation between indices of anxiety and depression, these factors were dissociable. This result is important because it allows clinicians to follow up with patients on specific symptoms of anxiety or depression and refer them to the appropriate empirically supported treatments. Given the overlap between the anxiety items on the HADS with posttraumatic stress disorder (PTSD) symptoms and the high comorbidity of PTSD in polytrauma samples, it is possible that a different factor structure may emerge if separate models were tested for participants with and without PTSD. Unfortunately, this analysis could not be completed given the current sample size and lack of definitive diagnosis of PTSD [45]. Future research with larger samples may improve the fit of the two-factor model.

The current HADS-A and HADS-D reliability estimates were very similar to the mean scores reported in a review study [26]. Additionally, this study demonstrated that the reliability estimates of the HADS scales were comparable to those of the BAI and BDI-2 in other studies, and the HADS was more reliable than the HAM-D
[8-11]. The HADS, however, contains one-third the items of the combined BAI and BDI-2. In settings where efficiency is stressed, such as TBI clinics, the shorter yet equally reliable HADS may be preferable for identifying symptoms of depression and anxiety that may warrant further evaluation by mental health professionals.

In this sample, on average, individuals reported moderate symptoms of anxiety and mild symptoms of depression. The majority of individuals indicated clinically meaningful scores of depression and/or anxiety. This result illustrates the clinical relevance of assessing symptoms of anxiety and depression in individuals undergoing evaluation for mTBI. Often, despite self-reported residual postconcussive symptomatology, the most salient symptoms among Veterans with histories of mTBI are emotional in nature [46]. For this reason, having an instrument such as the HADS is psychometrically useful for serial monitoring of these symptoms. Additionally, the factor structure of the HADS can give direction to clinicians and serve as a way to begin a conversation about mental health symptoms with patients who may not view their difficulties as emotional. Following this initial discussion in a VA polytrauma/TBI clinic, clinicians can refer Veterans for more targeted mental health services if warranted.

The HADS offers an efficient, structured method for gathering self-report data on anxiety and depression. The 
HADS is typically used in settings where assessment of anxiety and depression must be brief and data from the present sample, a VA polytrauma/TBI clinic, indicates that the anxiety and depression scales are of adequate reliability for brief assessments, screening, and most research purposes. Like all brief self-report instruments, the HADS has inherent weaknesses that must be compensated for by other assessment methods, such as clinical interview, behavioral observation, informant report, medical record review, and/or psychological testing. Anxiety and depression are common features in many mental and medical disorders; therefore, a thorough clinical history must be taken to determine whether symptoms are better accounted for by other conditions. Conversely, the items of the HADS relate to depression and anxiety in a general way and do not map directly onto any specific disorders in the fifth edition of the American Psychiatric Association's Diagnostic and Statistical Manual of Mental Disorders [47]. Another limitation is that the present study did not examine the convergent and divergent validity of the HADS. Future research can address this concern.

The brevity of the HADS may be regarded as a weakness because brief instruments typically yield lower reliability estimates than do longer instruments. It is a well-known phenomenon that, provided the items are of similar validity, longer instruments are more reliable than shorter ones. It can be argued, however, that longer selfreport measures have a point of diminishing returns, whereby little incremental information is gained from additional items. Although some may argue that longer instruments are better, this study has shown that despite its brevity, the HADS has psychometric properties comparable to longer instruments used to screen for anxiety and depression among Veterans in a polytrauma/TBI clinic.

\section{CONCLUSIONS}

The HADS is a brief, clinically useful measure of anxiety and depressive symptoms found to have very good reliability and two related, but distinctive, factors in a sample of young Veterans in a polytrauma/TBI clinic.

\section{ACKNOWLEDGMENTS}

\section{Author Contributions:}

Study concept and design: R. J. Spencer, L. L. Drag, L. A. Bieliauskas. Acquisition of data: L. L. Drag, P. H. Pangilinan.
Analysis and interpretation of data: L. Boxley, J. M. Flaherty, R. J. Spencer.

Drafting of manuscript: J. M. Flaherty, R. J. Spencer.

Critical revision of manuscript for important intellectual content:

L. Boxley, J. M. Flaherty, R. J. Spencer, L. L. Drag, P. H. Pangilinan, L. A. Bieliauskas.

Financial Disclosures: The authors have declared that no competing interests exist.

Funding/Support: This material was unfunded at the time of manuscript preparation.

Institutional Review: The parent study was approved by the Institutional Review Board at the VA Ann Arbor Healthcare System. Informed consent was not required because this was a retrospective study of deidentified data.

Participant Follow-Up: Because this was a retrospective study, participants will not be informed of the results. The authors have no plans to notify the study subjects of the publication of this article because of a lack of contact information.

\section{REFERENCES}

1. McCrae M. Mild traumatic brain injury and postconcussion syndrome: The new evidence base for diagnosis and treatment. New York (NY): Oxford University Press; 2008.

2. Kraal AZ, Waldron-Perrine B, Pangilinan PH, Bieliauskas LA. Affect and psychiatric symptoms in a Veteran polytrauma clinic. Rehabil Psychol. 2015;60(1):36-42.

[PMID:25528470]

http://dx.doi.org/10.1037/rep0000017

3. Waldron-Perrine B, Hennrick H, Spencer RJ, Pangilinan $\mathrm{PH}$, Bieliauskas LA. Postconcussive symptom report in polytrauma: Influence of mild traumatic brain injury and psychiatric distress. Mil Med. 2014;179(8):856-64.

[PMID:25102528]

http://dx.doi.org/10.7205/MILMED-D-13-00282

4. Zigmond AS, Snaith RP. The Hospital Anxiety and Depression Scale. Acta Psychiatr Scand. 1983;67(6):361-70.

[PMID:6880820]

http://dx.doi.org/10.1111/j.1600-0447.1983.tb09716.x

5. Beck AT, Steer RA. Beck Anxiety Inventory Manual. 2nd ed. San Antonio (TX): Psychological Corporation; 1993.

6. Beck AT, Steer RA. Manual for the revised Beck Depression Inventory. San Antonio (TX): Psychological Corporation; 1987.

7. Hamilton M. A rating scale for depression. J Neurol Neurosurg Psychiatry. 1960;23:56-62. [PMID:14399272]

http://dx.doi.org/10.1136/jnnp.23.1.56

8. Beck AT, Epstein N, Brown G, Steer RA. An inventory for measuring clinical anxiety: Psychometric properties. J Consult Clin Psychol. 1988;56(6):893-7.

[PMID:3204199]

http://dx.doi.org/10.1037/0022-006X.56.6.893

9. Fydrich T, Dowdall D, Chambless DL. Reliability and validity of the Beck Anxiety Inventory. J Anxiety Disord. 
1992;6(1):55-61.

http://dx.doi.org/10.1016/0887-6185(92)90026-4

10. Wang YP, Gorenstein C. Assessment of depression in medical patients: A systematic review of the utility of the Beck Depression Inventory-II. Clinics (Sao Paulo). 2013;68(9): 1274-87. [PMID:24141845] http://dx.doi.org/10.6061/clinics/2013(09)15

11. Trajković G, Starčević V, Latas M, Leštarević M, Ille T, Bukumirić Z, Marinković J. Reliability of the Hamilton Rating Scale for Depression: A meta-analysis over a period of 49 years. Psychiatry Res. 2011;189(1):1-9.

[PMID:21276619]

http://dx.doi.org/10.1016/j.psychres.2010.12.007

12. Crawford JR, Henry JD, Crombie C, Taylor EP. Normative data for the HADS from a large non-clinical sample. Br J Clin Psychol. 2001;40(4):429-34. [PMID:11760618] http://dx.doi.org/10.1348/014466501163904

13. Leung CM, Ho S, Kan CS, Hung CH, Chen CN. Evaluation of the Chinese version of the Hospital Anxiety and Depression Scale. A cross-cultural perspective. Int J Psychosom. 1993;40(1-4):29-34. [PMID:8070982]

14. Golden J, Conroy RM, O'Dwyer AM. Reliability and validity of the Hospital Anxiety and Depression Scale and the Beck Depression Inventory (Full and FastScreen scales) in detecting depression in persons with hepatitis $C$. J Affect Disord. 2007;100(1-3):265-9.

[PMID:17156850] http://dx.doi.org/10.1016/j.jad.2006.10.020

15. Gough K, Hudson P. Psychometric properties of the Hospital Anxiety and Depression Scale in family caregivers of palliative care patients. J Pain Symptom Manage. 2009; 37(5):797-806. [PMID:18789643]

http://dx.doi.org/10.1016/j.jpainsymman.2008.04.012

16. Martin CR, Thompson DR. Utility of the Hospital Anxiety and Depression Scale in patients with end-stage renal disease on continuous ambulatory peritoneal dialysis. Psychol Health Med. 1999;4:369-76. http://dx.doi.org/10.1080/135485099106117

17. Martin CR, Thompson DR. A psychometric evaluation of the Hospital Anxiety and Depression Scale in coronary care patients following acute myocardial infarction. Psychol Health Med. 2000;5:193-201.

http://dx.doi.org/10.1080/713690189

18. Martin CR, Tweed AE, Metcalfe MS. A psychometric evaluation of the Hospital Anxiety and Depression Scale in patients diagnosed with end-stage renal disease. Br J Clin Psychol. 2004;43(1):51-64. [PMID:15005906] http://dx.doi.org/10.1348/014466504772812968

19. McCue P, Buchanan T, Martin CR. Screening for psychological distress using Internet administration of the Hospital Anxiety and Depression Scale (HADS) in individuals with chronic fatigue syndrome. Br J Clin Psychol. 2006; 45(4):483-98. [PMID:17076959] http://dx.doi.org/10.1348/014466505X82379
20. McCue P, Martin C, Buchanan T, Rodgers J, Scholey A. An investigation into the psychometric properties of the Hospital Anxiety and Depression Scale in individuals with chronic fatigue syndrome. Psychol Health Med. 2003;8(4):425-39. [PMID:21974733] http://dx.doi.org/10.1080/1354850310001604568

21. Stafford L, Berk M, Jackson HJ. Validity of the Hospital Anxiety and Depression Scale and Patient Health Questionnaire-9 to screen for depression in patients with coronary artery disease. Gen Hosp Psychiatry. 2007;29(5): 417-24. [PMID:17888808] http://dx.doi.org/10.1016/j.genhosppsych.2007.06.005

22. Sukantarat KT, Williamson RC, Brett SJ. Psychological assessment of ICU survivors: A comparison between the Hospital Anxiety and Depression Scale and the Depression, Anxiety and Stress Scale. Anaesthesia. 2007;62(3):239-43. [PMID:17300300]

http://dx.doi.org/10.1111/j.1365-2044.2006.04948.x

23. Woolrich RA, Kennedy P, Tasiemski T. A preliminary psychometric evaluation of the Hospital Anxiety and Depression Scale (HADS) in 963 people living with a spinal cord injury. Psychol Health Med. 2006;11(1):80-90.

[PMID: 17129897] http://dx.doi.org/10.1080/13548500500294211

24. Cameron IM, Crawford JR, Lawton K, Reid IC. Psychometric comparison of PHQ-9 and HADS for measuring depression severity in primary care. Br J Gen Pract. 2008; 58(546):32-36. [PMID:18186994] http://dx.doi.org/10.3399/bjgp08X263794

25. Flint AJ, Rifat SL. Factor structure of the Hospital Anxiety and Depression Scale in older patients with major depression. Int J Geriatr Psychiatry. 2002;17(2):117-23.

[PMID:11813272]

http://dx.doi.org/10.1002/gps.535

26. Bjelland I, Dahl AA, Haug TT, Neckelmann D. The validity of the Hospital Anxiety and Depression Scale. An updated literature review. J Psychosom Res. 2002;52(2): 69-77. [PMID:11832252] http://dx.doi.org/10.1016/S0022-3999(01)00296-3

27. Dunbar M, Ford G, Hunt K, Der G. A confirmatory factor analysis of the Hospital Anxiety and Depression Scale: Comparing empirically and theoretically derived structures. Br J Clin Psychol. 2000;39(1):79-94.

[PMID:10789030]

http://dx.doi.org/10.1348/014466500163121

28. Whelan-Goodinson R, Ponsford J, Schönberger M. Validity of the Hospital Anxiety and Depression Scale to assess depression and anxiety following traumatic brain injury as compared with the Structured Clinical Interview for DSMIV. J Affect Disord. 2009;114(1-3):94-102. [PMID:18656266] http://dx.doi.org/10.1016/j.jad.2008.06.007

29. McHale M, Hendrikz J, Dann F, Kenardy J. Screening for depression in patients with diabetes mellitus. Psychosom 
Med. 2008;70(8):869-74. [PMID:18842744]

http://dx.doi.org/10.1097/PSY.0b013e318186dea9

30. Mondolo F, Jahanshahi M, Granà A, Biasutti E, Cacciatori E, Di Benedetto P. The validity of the Hospital Anxiety and Depression Scale and the Geriatric Depression Scale in Parkinson's disease. Behav Neurol. 2006;17(2):109-15. [PMID:16873922] http://dx.doi.org/10.1155/2006/136945

31. Mondolo F, Jahanshahi M, Granà $\mathrm{A}$, Biasutti $\mathrm{E}$, Cacciatori E, Di Benedetto P. Evaluation of anxiety in Parkinson's disease with some commonly used rating scales. Neurol Sci. 2007;28(5):270-5. [PMID:17972042] http://dx.doi.org/10.1007/s10072-007-0834-9

32. Poole NA, Morgan JF. Validity and reliability of the Hospital Anxiety and Depression Scale in a hypertrophic cardiomyopathy clinic: The HADS in a cardiomyopathy population. Gen Hosp Psychiatry. 2006;28(1):55-8. [PMID:16377366] http://dx.doi.org/10.1016/j.genhosppsych.2005.08.004

33. Walker J, Postma K, McHugh GS, Rush R, Coyle B, Strong V, Sharpe M. Performance of the Hospital Anxiety and Depression Scale as a screening tool for major depressive disorder in cancer patients. J Psychosom Res. 2007;63(1): 83-91. [PMID:17586341]

http://dx.doi.org/10.1016/j.jpsychores.2007.01.009

34. Dennis RE, Boddington SJ, Funnell NJ. Self-report measures of anxiety: Are they suitable for older adults? Aging Ment Health. 2007;11(6):668-77. [PMID:18074254] http://dx.doi.org/10.1080/13607860701529916

35. Nunnally JC. Psychometric theory. 2nd ed. New York (NY): McGraw-Hill; 1978.

36. Kline RB. Principles and practice of structural equation modeling. 3rd ed. New York (NY): The Guilford Press; 2011.

37. Drag LL, Spencer RJ, Walker SJ, Pangilinan PH, Bieliauskas LA. The contributions of self-reported injury characteristics and psychiatric symptoms to cognitive functioning in OEF/OIF veterans with mild traumatic brain injury. J Int Neuropsychol Soc. 2012;18(3):576-84. [PMID:22390876] http://dx.doi.org/10.1017/S1355617712000203

38. Spencer RJ, Axelrod BN, Drag LL, Waldron-Perrine B, Pangilinan PH, Bieliauskas LA. WAIS-IV reliable digit span is no more accurate than age corrected scaled score as an indicator of invalid performance in a Veteran sample undergoing evaluation for mTBI. Clin Neuropsychol. 2013; 27(8):1362-72. [PMID:24099142] http://dx.doi.org/10.1080/13854046.2013.845248

39. Waldron-Perrine B, McGuire AP, Spencer RJ, Drag LL, Pangilinan PH, Bieliauskas LA. The influence of sleep and mood on cognitive functioning among Veterans being evaluated for mild traumatic brain injury. Mil Med. 2012; 177(11):1293-301. [PMID:23198504] http://dx.doi.org/10.7205/MILMED-D-12-00169
40. Flaherty JM, Spencer RJ, Drag LL, Pangilinan PH, Bieliauskas LA. Limited usefulness of the Rey Fifteen-Item Test in detection of invalid performance in Veterans suspected of mild traumatic brain injury. Brain Inj. 2015; 29(1314):1630-4. [PMID:26513604] http://dx.doi.org/10.3109/02699052.2015.1075249

41. Benson J, Fleishman JA. The robustness of maximum likelihood and distribution free estimators to non-normality in confirmatory factor analysis. Qual Quant. 1994;28:117-36. http://dx.doi.org/10.1007/BF01102757

42. Browne MW, Cudeck R. Alternative ways of assessing model fit. In: Bollen KA, Long, JS, editors. Testing structural equation models. Newbury Park (CA): Sage; 1993. p. $136-62$.

43. Loehlin JC. Latent variable models. 3rd ed. Mahwah (NJ): Erlbaum; 1998.

44. Bentler PM, Bonett DG. Significance tests and goodness of fit in the analysis of covariance structures. Psychol Bull. 1980;88(3):588-606. http://dx.doi.org/10.1037/0033-2909.88.3.588

45. Lew HL, Otis JD, Tun C, Kerns RD, Clark ME, Cifu DX. Prevalence of chronic pain, posttraumatic stress disorder, and persistent postconcussive symptoms in OIF/OEF Veterans: Polytrauma clinical triad. J Rehabil Res Dev. 2009;46(6):697-702. [PMID:20104399] http://dx.doi.org/10.1682/JRRD.2009.01.0006

46. Bieliauskas LA, Drag LL, Spencer RJ. Long-term outcomes from traumatic brain injury. In: Koffler S, Morgan J, Marcopulos B, Greiffenstein MF, editors. A review of neuropsychology science and practice. Vol 2. New York (NY): Oxford University Press; 2015. p. 26-69.

47. American Psychiatric Association. Diagnostic and statistical manual of mental disorders. 5th ed. Washington (DC): American Psychiatric Association; 2013.

Submitted for publication May 20, 2015. Accepted in revised form December 16, 2015.

This article and any supplementary material should be cited as follows:

Boxley L, Flaherty JM, Spencer RJ, Drag LL, Pangilinan $\mathrm{PH}$, Bieliauskas LA. Reliability and factor structure of the Hospital Anxiety and Depression Scale in a polytrauma clinic. J Rehabil Res Dev. 2016;53(6):873-80. http://dx.doi.org/10.1682/JRRD.2015.05.0088

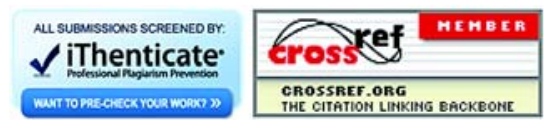

